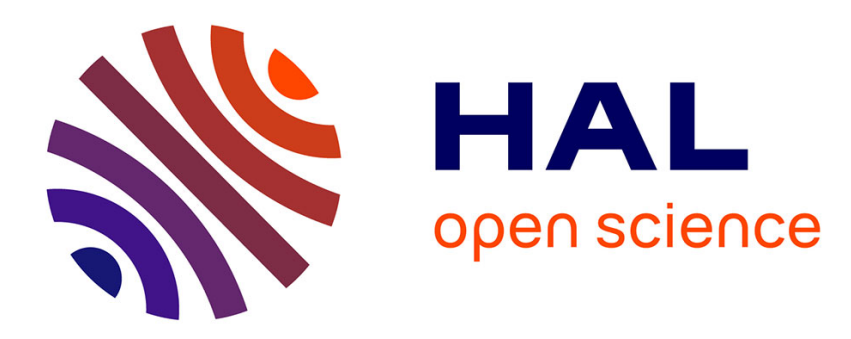

\title{
L'exposition de soi sur Internet: un souci d'être au-delà du paraître
}

Francis Jauréguiberry

\section{To cite this version:}

Francis Jauréguiberry. L'exposition de soi sur Internet: un souci d'être au-delà du paraître. N. Aubert et C. Haroche. Les tyrannies de la visibilité, Érès, pp.131-144, 2011, 10.3917/eres.auber.2011.01.0131 . hal-01669055

\section{HAL Id: hal-01669055 https://hal.science/hal-01669055}

Submitted on 21 Jan 2018

HAL is a multi-disciplinary open access archive for the deposit and dissemination of scientific research documents, whether they are published or not. The documents may come from teaching and research institutions in France or abroad, or from public or private research centers.
L'archive ouverte pluridisciplinaire HAL, est destinée au dépôt et à la diffusion de documents scientifiques de niveau recherche, publiés ou non, émanant des établissements d'enseignement et de recherche français ou étrangers, des laboratoires publics ou privés. 
Paru dans Les tyrannies de la visibilité (éds. N. Auber et C. Haroche), Toulouse, Érès, 2011, pp. 131-144.

\title{
L'exposition de soi sur Internet : un souci d'être au-delà du paraître
}

\author{
Francis Jauréguiberry
}

\section{Introduction}

En quelques années seulement, il est devenu quasiment impossible de ne pas apparaître sur Internet. Il est en effet de plus en plus difficile d'appartenir à une entreprise, une organisation ou une institution sans que celle-ci ne fasse une brève présentation de ses collaborateurs sur son site. De même, avoir une activité publique se double immanquablement par une référence sur le web. Par exemple, le simple fait de participer à ce colloque nous vaut au moins deux ou trois références sur le web... La capacité des systèmes experts à tracer des profils de consommateurs à partir des sites fréquentés et à croiser l'ensemble des données individuelles laissées sur Internet offre une image de chacun de plus en plus détaillée et précise $^{1}$. Ce phénomène de duplication automatique de soi sur Internet est tel que, dans l'environnement d'injonction général à la visibilité bien cerné lors de ce colloque, il devient suspect de ne pas apparaître sur Internet, de la même façon qu'il est désormais reproché à certains de ne pas répondre immédiatement au téléphone ou à un courrier électronique.

Il faut donc être très attentif et de plus en plus rusé pour échapper à cette grande indexation de soi sur Internet. Mais le veut-on vraiment ? Pour l'instant, toutes les enquêtes que j'ai pu mener ou dont j'ai pu prendre connaissance sur ce sujet ont montré que la quasitotalité des personnes interrogées étaient plutôt flattées d'être sur Internet, l'attitude la plus négative étant l'indifférence. Les conduites défensives visant à instaurer des formes de déconnexions partielles, éphémères et changeantes, synonymes de discontinuité, de mise à distance et d'opacité là où les systèmes poussent à la continuité, à l'immédiat et à la transparence sont encore très rares. Au contraire, les conduites volontaires visant à être vu, écouté, répertorié, cité et si possible adulé sur Internet ont explosé ces dernières années. Aujourd'hui même, des dizaines de milliers d'individus en France, des millions de par le monde, vont parler d'eux en alimentant leur blog ou en commentant celui d'un autre, en complétant leur profil sur un réseau social, en affichant leurs photos ou en mettant en ligne leurs vidéos. Ils y parleront de leurs expériences, de leurs peines, de leurs soucis, ou de leurs attentes. Mais aussi de leurs passions pour un genre musical, un auteur, un chanteur. Ils feront part de leurs commentaires sur un fait d'actualité, un problème social ou une déclaration politique. Et, en retour, des dizaines ou des milliers (selon les sites) d'individus les liront et réagiront peut-être. Cette mise en exposition de soi, de ses pensées, de ses fantasmes ou de ses productions ne relève pas de la contrainte, d'une «tyrannie de la visibilité » comme il a été dit, mais plutôt d'une « servitude volontaire » à s'exposer. C'est de ce phénomène dynamique et volontaire dont il sera question ici.

\footnotetext{
${ }^{1}$ Dans un article assez décrié, un journaliste a récemment reconstitué la vie d'un inconnu à partir des traces que ce dernier avait, volontairement ou pas, laissées sur Internet. Ce n'étaient pas seulement les statut, adresse et numéro de téléphone de cet inconnu qui étaient révélés, mais aussi son emploi du temps, ses goûts, ses relations amoureuses, ses amis, les endroits qu'il fréquentait, etc. (Raphaël Meltz, «Marc L*** », in Le Tigre, volume 28, 2008, pp. 36-37). Sur ce thème, voir Bradwell P. et Gallagher N. (2007).
} 


\section{1- L'hypothèse du cyber-narcissisme}

Une première lecture de cette mise en visibilité de soi sur Internet, assez courante semble-t-il (et en tout cas dominante lors des deux premiers jours de ce colloque), consiste à assimiler cette démarche à une simple exposition de soi narcissique. Exposition donc d'un individu qui se dévoile sur la toile afin d'obtenir une validation publique et positive de luimême. L'apparente obsession des amateurs de réseaux sociaux (SNS : Social Networked Sites) à mesurer leur audience, à la repérer et à la cataloguer semble aller dans le sens de cette première lecture, celle d'un cyber-narcissisme ${ }^{2}$. Ce qui est recherché, c'est la célébrité, la notoriété; c'est d'être lu, vu et écouté par le plus grand nombre. Le recours quasi systématique à des instruments comme Xiti ou Webstats, qui permettent de comptabiliser et de localiser les visites, trahit bien ce désir d'audience. Les plateformes hébergeant les blogs, exposant des photos personnelles (par exemple Flickr), donnant à visionner des vidéos privées (par exemple DailyMotion) ou diffusant des compositions musicales inédites (par exemple MySpace) proposent toujours un compteur qui figure sur le premier écran et la plupart du temps un classement de popularité. L'obsession semble être ici : combien de personnes m'ont lu, vu, écouté ? Et si le chiffre d'audience n'est pas trop mauvais, alors démonstration est faite: j'existe bien aux yeux de tous ces visiteurs et je suis donc, finalement, célèbre.

Plusieurs intervenants ont bien rappelé la généalogie et le développement de l'injonction prométhéenne lancée à l'individu contemporain de se produire lui-même et à réussir sa vie. Alors que, jusqu'à peu, les normes sociales disciplinaient l'individu et le portaient à habiter sa condition (produisant conformité ou, au contraire, révolte), elles l'assignent aujourd'hui à devenir lui-même sans autre référence que sa propre volonté. Alors que, jusqu'au seuil des années 1970, un futur chargé d'espoir pouvait collectivement être entrevu à travers les idéologies d'émancipations, l'actuelle désaffection envers les utopies sociales laisse place à un présent qu'il s'agit de conquérir personnellement à partir de ses propres ressources. On n'espère plus collectivement dans le futur : il faut réussir personnellement dans le présent. L'individu est mis en face de ses propres réalisations. La nécessité de réussite se double d'un indispensable souci de reconnaissance. Et il s'agit certainement là d'une des motivations de ceux qui s'exposent sur le Net. Les blogs comme les réseaux sociaux du Net peuvent être vécus comme un espace aisé de validation d'un soi à la fois producteur et inquiet de lui-même. La réalité de ce type de conduite est patente : il suffit de se rendre sur n'importe quelle plateforme du web pour s'en rendre compte.

\section{2- Le Networking instrumental}

Une autre lecture du même phénomène consiste à mettre l'accent sur son aspect utilitaire et instrumental. La présentation de soi (sous la forme d'un profil, de critères ou de productions choisies) est dans ce cas moins faite dans un but de reconnaissance et d'attestation de soi, que jouée comme ressource afin d'obtenir des bénéfices matériels ou symboliques. La reconnaissance n'est plus un but, mais un moyen. Qu'est-ce que ce je suis peut m'apporter de plus si je l'expose sur Internet? Le succès de certaines plateformes du web relationnel est parfaitement révélateur de ce souci de reconnaissance intéressée. Le but visé est de nouer des relations utiles pour sa carrière professionnelle (par exemple sur Linkedin), d'obtenir des rendez-vous amoureux (par exemple sur Meetic), de profiter des avantages d'un voisinage partagé (par exemple sur Peuplade) ou encore de décrocher des contrats de promotion ou des tournées musicales (par exemple sur MySpace). Dans chaque cas, il y a déclinaison et mise en publicité de soi afin de parvenir à des fins. Il s'agit de paraître souvent et si possible mieux afin d'avoir plus. En cas d'hésitation, les moins

\footnotetext{
${ }^{2}$ Pour une présentation synthétique des travaux portant sur les réseaux sociaux sur Internet, voir Danah Boyd et
} Nicole Ellison (2008). 
performants peuvent d'ores et déjà faire appel à des coachs ou à des sites spécialisés (par exemple Ziki) pour les aider à se présenter sur le Net sous leur meilleur jour...

De leur côté, les «amis », connaissances et profils sont triés selon un ensemble de critères permettant à l'internaute de mesurer leur plus ou moins grande capacité à répondre favorablement à ses attentes. Il s'agit de repérer, évaluer et cataloguer les membres du réseau selon une logique purement utilitaire. Qui peut m'amener quoi et dans quelle condition? Cette logique d'action ne semble pas s'embarrasser d'états d'âme, de tourments moraux et encore moins de sentiments de générosité ou de compassion. L'altruisme et le partage, lorsqu'ils se donnent à voir, ne sont avancés que comme «mises » dans le jeu stratégique avec l'espoir de les récupérer décuplées. Autrui n'est finalement recherché qu'en ce qu'il est source de gains potentiels.

Zapper est l'action spécifique qui résume le mieux l'attitude de l'internaute dans cette recherche de bénéfices possibles. Il s'agit pour lui d'adopter la conduite la plus agile possible, d'aller rapidement d'un site à un autre, d'un interlocuteur à un autre et d'une information à une autre afin d'en évaluer les potentialités. Zappant, parmi les centaines d'interlocuteurs possibles, de leurs descriptions physiques à leurs centres d'intérêt, de leurs autoportraits à leurs profils identitaires, l'internaute cherche à se faire une idée de qui pourrait s'avérer le plus apte à combler ses attentes. Se basant sur un ensemble de critères objectifs de catégorisation ou de déclinaisons subjectives de goûts, sentiments ou attitudes, il est à la recherche du meilleur choix possible. Les sites de rencontre (par exemple Meetic) poussent cette logique encore plus loin: les internautes se servent de moteurs de recherche pour sélectionner automatiquement un certain nombre de profils à partir de critères physiques, de statuts sociaux et de catégories descriptives en termes de goûts ou d'activités (Illouz, 2006). Une période de zapping intense est le préalable à de potentiels choix de rencontres, le dévoilement (des pseudonymes aux personnes réelles et donc à un rendez-vous en face à face) se faisant ensuite progressivement par un processus de mise en confiance réciproque (Chaulet, 2006).

Le type de communication qui correspond à cette approche du réel est presque toujours utilitaire, instrumental et informatif. Le calcul et le jeu définissent le mieux les actions des uns envers les autres, chacun étant considéré par chacun comme potentielle ressource ou cible avec lesquelles il faut «faire des coups ». Dans ce type de démarche, chacun est à la fois metteur en scène de lui-même et spectateur des représentations des autres. Toutefois, la distance entre l'identité réelle et l'identité numérique ne saurait être trop importante, le but étant in fine d'obtenir quelque chose dans la vie réelle, en particulier dans une situation de présence physique. Les critères et profils déclinés doivent donc être concrètement vérifiables. Cela diffère grandement d'un autre type de conduites observables sur le Net : l'essai de soi comme un autre.

\section{3- L'essai de soi comme un autre}

Une distinction s'impose entre les sites où les individus s'exposent tels qu'ils pensent être réellement dans leur vie quotidienne, et les sites où la dissimulation, le déguisement ou le travestissement identitaire sont monnaie courante. Dans un essai de typologie des plateformes relationnelles du web, Dominique Cardon (2008) montre combien «chaque plateforme propose une politique de la visibilité spécifique et cette diversité permet aux utilisateurs de jouer leur identité sur des registres différents ». Il distribue en particulier les plateformes sur un axe selon la plus ou moins grande latitude identitaire qu'elles autorisent. À une extrémité, celles qui exigent que noms, statuts et adresses e-mail soient publics et qui ne laissent aucun espace à la dissimulation et l'imagination identitaire. À l'opposée, celles qui imposent l'anonymat et le port d'un masque sous la forme de pseudonymes, et sur lesquelles tout emprunt et travestissement identitaire est donc possible. Entre ces deux extrêmes se situe 
l'essentiel de ce qui se passe sur le Net avec de subtils jeux de masque permettant à chacun de contrôler plus ou moins finement ce qu'il veut laisser paraître ou donner à voir de lui-même.

Il est impossible de parler de l'exposition de soi sur Internet sans évoquer cette particularité que les plateformes qui se situent du côté de l'anonymat permettent : l'exhibition de soi sous des traits qui ne correspondent que très partiellement, si ce n'est pas du tout, à son identité civile réelle et à ses statuts reconnus dans la vie quotidienne. Le dispositif technique de certaines plateformes permet à l'individu de "s'essayer » à différentes formes de soi qu'il teste avec l'intention d'expérimenter «l'effet que ça fait». Une foule d'emprunts identitaires est ainsi désormais possible sur Internet. Par exemple, une jeune femme de vingt ans peut parfaitement apparaître sous les traits d'un homme de quarante ans. Dans sa vie quotidienne, elle a bien sûr tout loisir de s'imaginer être un homme. Mais le réel lui renvoie son image de femme. Sur Internet, il y a comme un début de réalisation de son fantasme dans la mesure où son identité d'emprunt est reconnue comme telle par ses interlocuteurs. On s'adresse à cette jeune femme comme à un homme : là réside l'essentiel. Il ne s'agit plus simplement d'une identité fantasmée, produit d'un rêve éveillé, mais bien d'une forme de réalité partagée. La tangibilité de ce soi d'emprunt réside en effet dans le fait qu'il est vécu comme une réalité par autrui, quand bien même cet autrui-là serait-il lui-même la projection d'un autre fantasme. Ce qui intéresse l'internaute qui manipule son identité, ce n'est pas tant d'habiter un personnage que d'être reconnu comme tel en communiquant.

Dans un précédent article (Jauréguiberry, 2004), je décrivais les dérives auxquelles pouvait mener un tel travestissement de soi. Partant de la présentation que fait Alain Ehrenberg (1998) de la dépression comme «pathologie de l'insuffisance » s'inscrivant la «déception » et la «fatigue» de l'individu contemporain qui ne parvient pas à vivre l'idéal qu'il s'est lui-même fixé, je présentais la manipulation de soi sur Internet comme une façon d'échapper à cette conscience malheureuse de n'être que soi-même. Selon cette optique, la manipulation de soi vise à combler le vide que connaît l'individu hypermoderne entre la conception surévaluée qu'il se fait de lui-même et la perception de sa réelle condition. Plutôt que de se désespérer du fait de n'être "que lui-même », l'individu va se construire à peu de frais un moi beaucoup plus conforme à ses désirs par le biais d'emprunts identitaires valorisants ou gratifiants. Mais, dans son écoute constante envers lui-même, le moi du «manipulateur de soi » risque alors de ne plus être que quête d'émois. Ce qui est recherché, c'est un recouvrement de soi par son fantasme, c'est l'oubli de la distance critique qu'introduit le je introspectif et donc des tensions, limites, et souvent frustrations que cette distance révèle. Goûtant sans retenue les délices de la reconnaissance de ses attentes par le réseau, l'individu court alors le risque de s'enfermer dans une pratique compulsive d'Internet.

Mais, autant cette conséquence extrême à laquelle peut conduire l'essai de soi comme un autre sur Internet peut être inquiétante, autant une autre issue peut être considérée avec un certain optimisme. Elle conduit à interpréter ce décalage identitaire comme l'opportunité saisie par certains internautes pour échapper aux images trop restrictives d'eux-mêmes que leur renvoie la société sous la forme de statuts, d'assignations socioculturelles et de rôles incontournables. Leur identité numérique décalée révèle, sans doute maladroitement, d'un désir d'exister «autrement». Non pas pour s'y perdre, pour fuir la réalité, mais pour mieux y tester leur autonomie et leur aspiration à la liberté. L'emprunt, au moins partiel, d'identités fictives serait dans ce cas pour l'internaute une façon de se dédouaner des contraintes qui pèsent sur lui en tant qu'objet social et d'expérimenter, de façon détournée, son aspiration à être un sujet. Ce ne serait pas tant la réalisation de soi comme un autre qui serait alors recherchée que la capacité à s'étonner d'un décalage à soi-même.

On peut en effet considérer que les identités numériques sont tout autant des bases de résistance que des points de fuite, tout autant des actes créatifs que des abandons identitaires. La manipulation de soi par un individu sur Internet peut donc aussi (et pas seulement, bien 
évidemment) être lue aussi comme l'extériorisation transgressive de son exigence d'être un sujet créatif au-delà des déterminations, statuts et rôles qui le freinent socialement. Même si les changements sociaux dus à une telle pratique sont pour l'heure très faibles, une lecture optimiste de cette perspective conduit à penser que l'expérience d'identités numériques décalées peut fonctionner comme espace de recadrage de soi et des autres dans une attitude plus ouverte et créatrice. Le fait d'habiter ainsi une ou des altérités pourrait alors aider à mieux écouter, à mieux comprendre les autres dans leur complexité, ceux-ci cessant alors d'être appréhendés comme des objets pour être abordés comme des sujets avec les tensions et les faiblesses, mais aussi avec la force et l'inventivité que cela suppose. Cette dynamique est particulièrement observable sur les blogs.

\section{4- L'hypothèse du déploiement de soi}

Les emprunts identitaires, présents dès les débuts d'Internet (Turkle, 1997) et même avant sur le Minitel (Marchand et al, 1987), ne sont pas de mise sur les blogs. Il est évidemment possible, et c'est souvent ce qui arrive, d'y " améliorer» certains traits de son identité réelle. Mais pas trop car, pour fonctionner, le dévoilement sur un blog doit être sincère. Le recours quasi général (en dehors des blogs de personnalités ou d'institutions) à des pseudonymes ne signifie pas dissimulation, mais au contraire masque permettant une forme d'authenticité. Il ne s'agit pas pour le blogueur d'habiter un autre personnage que lui, mais de donner à partager une passion, un savoir ou plus simplement une réflexion, un questionnement ou un étonnement. Lorsqu'on parcourt les listings de blogs, on découvre un foisonnement incroyable des centres d'intérêt exposés avec patience et la plupart du temps avec pertinence et goût qui trahissent les personnalités, passions et pensées réelles de ceux qui les tiennent. C'est aussi ce que relève Annabelle Klein (2007, p. 213) en conclusion de l'ouvrage qu'elle a dirigé sur les blogs : «La construction de mécanismes identitaires dans la blogosphère est particulièrement intéressante car, contrairement à d'autres dispositifs de communication sur Internet tels que les chats, sites de rencontre, jeux de rôle, etc., le "jeu" ne se situe absolument pas du côté, généralement relevé, du faux, du mensonge, du fictif, de l'expérimentation à être autre, du jeu à devenir ce qu'on n'est pas ».

Bien sûr, les blogueurs sont aussi à la recherche de reconnaissance et parfois de notoriété, en particulier lorsqu'il s'agit de blogs «d'experts ». Certains sont ainsi devenus de véritables vedettes, presque incontournables sur certains sujets, ayant parfois plus de succès que des spécialistes jusqu'alors reconnus dans les réseaux classiques. Mais les vedettes sont peu nombreuses : selon une étude approfondie du MIT, seul $1 \%$ des blogs dépasse la centaine de visiteurs par jour. Si tous les blogueurs sont attentifs au nombre de visites, ce n'est pas tant pour faire du chiffre que pour savoir, tout simplement, s'ils sont lus. Qu'il y ait ensuite vingt ou cent lecteurs fait moins la différence que la qualité des commentaires qu'ils reçoivent. Ceux-ci font intégralement partie du blog et fonctionnent comme un gage de reconnaissance et de validation (Allard et Vandenberghe, 2003).

Lorsqu'on prend le temps d'écouter les blogueurs et de les suivre dans leur production, on s'aperçoit vite qu'au-delà du paraître, c'est bien d'une question d'être dont il s'agit ${ }^{3}$. Dit rapidement, le blog leur permet de déployer une part d'eux-mêmes que leur position sociale rend difficile à vivre. Le blog leur permet d'habiter et de développer des pans de leur personnalité jusqu'alors floués, contrariés, voire interdits par leur environnement social. Mais, contrairement aux emprunts identitaires précédemment évoqués qui n'étaient que purs fantasmes, il s'agit là de pans identitaires authentiques aux yeux du blogueur. Un art, une pratique, une intuition, cultivés dans l'intimité faute d'un espace public d'expression trouve

\footnotetext{
${ }^{3}$ Ces réflexions reposent sur un travail actuellement en cours mené auprès de 18 (pour l'instant) bloggeurs. Les entretiens ont tous commencé par un ou plusieurs commentaires puis par échange d'e-mails. Parallèlement, un suivi au fil de l'eau de plusieurs autres blogs est mené.
} 
ici un lieu de déploiement. Ainsi ce petit vigneron, dans un petit village, qui tient un blog de poésie. Ses poèmes étaient toujours restés secrets. Sauf une fois, il y a longtemps de cela, lorsque, jeune homme, il en avait montré certains à des amis qui s'étaient moqué de lui. Depuis, il n'en avait plus jamais reparlé. Jusqu'à ce qu'il décide, sous pseudonyme, d'en publier quelques-uns sur un blog sommaire. Et grand fut son étonnement de voir qu'une puis deux, puis (au fur et à mesure où il échangeait avec ses premiers interlocuteurs) plusieurs personnes inconnues commentèrent ses écrits. Des échanges fructueux se nouèrent, débouchant dans certains cas sur de véritables « camaraderies numériques ». Le petit vigneron n'a pourtant révélé son identité à aucun de ses interlocuteurs. Ce qui l'intéresse n'est pas d'avoir des centaines de lecteurs et d'être célèbre, mais d'être reconnu dans un pan de sa personnalité créative qu'il estime essentielle. Bien sûr, sans Internet, il aurait sans doute continué d'écrire, mais avec une certaine souffrance : celle, paradoxale en la matière, de devoir se taire.

De façon très imagée, un blogueur m'a dit «qu'écrire (sur son blog) ce que la vie lui inspirait le faisait inspirer plus profondément la vie». Un autre : «C'est comme si la vie était plus riche». Et un autre encore : «Si on me supprimait mon blog, ce serait comme un arrachement d'une partie de ma vie, peut être celle qui m'apporte le plus en ce moment». Dans ces cas, tenir un blog n'est donc pas anecdotique, mais renvoie à quelque chose de vital. Il semble, qu'à travers les blogs, soit expérimenté de façon inédite ce que le processus de subjectivation des individus ne cesse de produire : un décalage constant aux définitions de soi comme objet social. Selon cette optique, l'anonymat des blogueurs sert moins à se cacher qu'à échapper à la catégorisation sociale dont ils sont l'objet. Le masque tient à distance ce qui socialement contraint et empêche souvent la subjectivité et la créativité individuelle de se déployer ${ }^{4}$. «L'intérêt, écrit un blogueur, c'est qu'on est lu pour ce qu'on écrit ou vu pour ce que l'on a créé (et non pas en fonction de sa position sociale). »Dans la vie réelle, la catégorisation sociale classe, oriente et souvent exclue. Ici, pas de biographie en dehors de ce que le blogueur estime devoir dévoiler de lui. N'apparaît que ce qui lui importe et qui ne trouve pas ou pas suffisamment place dans le quotidien ${ }^{5}$.

Le sentiment d'exister en dehors ou plutôt en plus de son statut professionnel, de son appartenance familiale et de ses assignations sociales est la base même de l'expérience de soi comme sujet, c'est-à-dire comme individu autonome et capable de créativité. La confrontation de ce sentiment avec un environnement social réducteur et contraignant peut conduire à un exil intérieur, synonyme de démission résignée ou d'acceptation meurtrie. Cette tension, qui est au fondement même des modalités existentielles de la modernité, se trouve multipliée chez l'individu contemporain (Martuccelli, 2002). La mise à distance du monde, qui a accompagné son objectivation et sa rationalisation, a permis de le rendre plus maitrisable et contrôlable. Comme le souligne Giddens (1994), le développement technologique et organisationnel a délivré l'individu des dangers et craintes situationnelles d'autrefois. Mais l'approfondissement du caractère objectif de la vie, en désamorçant les réponses sociales «enchantées » qu'apportaient mythes et traditions au questionnement existentiel, est aussi celui de l'insécurité ontologique. Celle-ci conduit l'individu à un nécessaire travail de construction de son existence de telle sorte qu'elle puisse avoir un sens à ses propres yeux. En

\footnotetext{
${ }^{4}$ En dehors de cet anonymat, il a été expérimentalement prouvé que l'écran tient à distance et donc permet une expression libérée de pans de sa personnalité. S'appuyant sur la définition que Carl Rogers donnait du « vrai soi » (true self) comme "part de soi que l'on pense être mais qui ne se donne pas à expérimenter dans la vie sociale », John Bargh, Katelyn McKenna et Grainne Fitzsimons (2002) ont ainsi montré qu'il était plus aisé d'exprimer cette part de soi par Internet qu'en face à face.

${ }^{5}$ Il existe cependant une catégorie de blogs dont le but est de maintenir des relations suivies avec des personnes affectivement proches. Il s'agit de tenir au courant sa famille et ses amis proches en postant de temps en temps des nouvelles, des photos ou vidéos, chaque blog renvoyant à un nombre limités d'autre blogs qui forment ensemble un réseau finalement privé (sur ce thème, voir Michael Stefanone et Chyng-Yang Jang (2007).
} 
somme, en l'absence d'un traitement social, l'individu doit trouver en lui-même le sens de son existence et surtout en expérimenter l'efficience sous la forme d'un contentement et d'un sentiment de vérité. La radicalisation instrumentale du réel se traduit chez l'individu par un besoin de préserver sa propre subjectivité sous une forme d'authenticité de soi à soi et de soi aux autres. Et l'on peut faire l'hypothèse que les blogs sont aussi un espace inédit permettant de vivre cette aspiration.

\section{De nouveaux liens}

Le blog est une sorte d'anti-portable ${ }^{6}$. Avec le portable, en dehors des appels purement instrumentaux vers des services ou des réseaux professionnels, l'essentiel des appels est destiné à très peu de personnes affectivement proches. Les portables servent à instaurer une espèce de liens permanents à la fois affectuels et organisationnels avec la famille et les amis (Jauréguiberry, 2003). Mais, dans les espaces publics, ces appels créent davantage de distance avec les inconnus physiquement proches. Dans une file d'attente, par exemple, pour ne pas subir de temps mort, on téléphone. Cet appel approfondit des liens déjà forts (avec la personne affectivement proche, mais géographiquement lointaine), mais amoindrit des liens déjà faibles (avec les personnes physiquement proches, mais affectivement lointaines). Liens sans doute faibles, mais essentiels à la formation du goût d'être en société et à la facilité de s'y mouvoir de façon aimable. Le blog est au contraire une ouverture constante à ces liens faibles, mais pouvant, au gré des circonstances, s'avérer importants. Le portable recentre sur le connu et le privé, le blog ouvre sur l'inconnu et le public.

Une lecture superficielle du phénomène blog l'a trop vite cataloguée comme exhibition ou pure dilatation d'un ego en mal de reconnaissance. Si l'on part de l'idéal type habermasien de l'espace public, force est effectivement de reconnaître que peu de blogs sont des lieux d'exposition et d'échange d'arguments fondés en raison. Mais l'espace public est aussi un espace d'échange de subjectivités, de sensibilités et de réalisations personnelles. Et il serait bien réducteur de systématiquement qualifier d'exhibition le fait de rendre public ses sentiments, ses émotions, ses intuitions, craintes ou émerveillements. Au contraire, il est remarquable de voir comment un blog, pour peu évidemment qu'il suscite des réactions, permet de passer d'une intuition individuelle à une idée discutée ou d'une petite remarque à un argument débattu. Le dispositif même des blogs, par le biais des commentaires, des hyperliens, des blogrolls et des tracbacks, crée en un second temps, des zones de contagion et d'affinités faisant, là aussi, lien, réseaux, échanges. Ce qui est attendu, ce sont les réactions d'autres sujets, d'autres «je » à la recherche d'eux-mêmes et qui ont envie de se construire dans une relation certes faible, mais sincère aux autres. Le succès des blogs pointe autant le désir individuel de reconnaissance que l'immense besoin de lien, d'écoute et d'échange que la vie « réelle» ne comble pas.

On pourrait même inverser la proposition qui réduit les blogs à n'être que des lieux matérialisant l'éclatement du social en autant d'egos mus pas un seul désir de reconnaissance, en notant que ce qui, au départ, est complètement individuel, intime et subjectif, peut, par sa seule exposition sur un blog, devenir objet de débat et donc créer du lien social. Le caractère public des posts et les commentaires déprivatisent l'échange, problématisent les intuitions, suscitent le débat et, finalement, sont producteurs de lien social. La particularité de ces liens noués sur les blogs est qu'ils ne ligotent pas. Ils peuvent à la fois être intenses et distendus. L'anonymat délivre des contraintes statutaires et permet l'expression des choses jusqu'alors tues. Au pire, un blogueur qui s'y sentirait enfermé peut déserter son blog du jour au lendemain.

\footnotetext{
${ }^{6}$ Tout au moins tant que les potables sont utilisés comme simples téléphones. Tout change à partir du moment où ils deviennent aussi des terminaux Internet.
} 


\section{Conclusion}

Que ce soit l'exposition de soi dans l'espérance d'une reconnaissance, la présentation de soi comme moyen d'obtenir des avantages ou la fuite de soi dans une identité fictive, ces trois lectures rendent incontestablement compte du phénomène chaque jour grandissant de la mise en visibilité de soi sur Internet. Mais elles n'en épuisent pas la signification. En particulier, les blogueurs ordinaires ne cherchent pas tous à être extraordinaires. Ils cherchent plus modestement à être eux-mêmes dans leur pluralité identitaire et dans leur capacité créatrice que leur environnement immédiat frustre trop souvent. On peut certes lire leur expression sur Internet comme un geste mégalomaniaque, égocentrique ou intéressé, mais il faut aussi, et peut-être surtout, y voir une volonté d'être dans sa complexité et une aspiration à créer selon ses talents. En cela les blogs sont révélateurs du fantastique potentiel de création, de dons et d'écoute que recèlent nos sociétés au-delà de la vision instrumentale et intéressée qui semble les guider.

\section{Références}

Allard L. et VANDENBERGHE F. (2003), «Express yourself! Les pages perso », Réseaux $\mathrm{n}^{\circ} 117, \mathrm{pp} .191-219$.

Bargh J., McKenna K. Et Fitzsimons G. (2002), «Can You See the Real Me ? Activation and Expression of the "True Self" on the Internet », Journal of Social Issues, vol. 58, $\mathrm{n}^{\circ} 1$, pp. 33-48.

BoYD D. et Ellison N. (2008), « Social Network Sites : Definition, History and Scholarship », Journal of Computer-Mediated Communication, vol. 13, n 1, pp. 210-230.

BRADWell P. ET Gallagher N. (2007), For Your Information. The new politics of personal information, Demos, London.

CARDON D. (2008), http://www.internetactu.net/2008/02/01/le-design-de-la-visibilite-unessai-de-typologie-du-web-20/ (consulté le 5/05/2008).

CHAulet J. (2006), « Les différents visages de l'information à l'heure de la généralisation des communications médiatisées », in Actes du colloque Interroger la société de l'information, Montréal, AISLF GT 13, pp. 205-212.

EHRENBERG A. (1998), La fatigue d'être soi, Paris, Odile Jacob.

GidDEn A. (1994), Les conséquences de la modernité, Paris, l'Harmattan.

ILlOUZ E. (2006), Réseaux amoureux sur Internet, Réseaux, n 138, pp. 243-268.

JAUREGUIBERRY F. (2003), Les branchés du portable. Sociologie des usages, Paris, PUF.

JAUREGUIBERRY F. (2004), «Hypermodernité et manipulation de soi, in L'individu hypermoderne (éd. AUBERT N .), Toulouse, Érès, pp. 155-168.

KLEIN A. (dir.) (2007), Objectif Blogs! Exploration dynamique de la blogosphère, Paris, l'Harmattan.

MaRChAND M. ET Le SPES (1987), Les paradis informationnels. Du Minitel aux services de communication du futur, Paris, Masson.

MARTUCCELli D. (2002), Grammaires de l'individu, Paris, Gallimard.

STEFANONE M. ET JANG C-Y. (2007), Writing for Friends and Family : Interpersonal Nature of Blogs, Journal of Computer-Mediated Communication, vol. 13, $\mathrm{n}^{\circ}$ 1, pp. 123-140. 
TURKLE S. (1997), Life on the Screen. Identity in the Age of the Internet, New York, Touchstone. 